\title{
A new subgenus and species of the genus Pseudobathystomus Belokobylskij (Hymenoptera: Braconidae: Rhyssalinae) from the Canary Islands
}

\author{
Sergey A. Belokobylskij \& Martti Koponen
}

Belokobylskij, S. A. \& Koponen, M. 2004: A new subgenus and species of the genus Pseudobathystomus Belokobylskij (Hymenoptera: Braconidae: Rhyssalinae) from the Canary Islands. - Entomol. Fennica 15: 225-230.

Anew subgenus Atlantobathystomus subgen. n. of the genus Pseudobathystomus Belokobylskij is described and illustrated for the new species $P$. macaronesius $\mathrm{sp}$. n. from Tenerife. A key to all known species of this genus is given.

Sergey A. Belokobylskij, Museum and Institute of Zoology PAN, Wilcza 64, Warsaw, Poland.E-mail: hymenopt@zin.ru; sb@zin.ru

Martti Koponen, Department of Applied Biology, PB 27, FIN-00014 Helsinki University, Finland.E-mail: martti.a.koponen@helsinki.fi

Received 14 November 2003, accepted 26 August 2004

\section{Introduction}

The subfamily Rhyssalinae was established by Foerster (1862), but has been considered a synonym of Exothecinae or Rogadinae for many years (Shenefelt 1975). After a study of internal morphology, mainly the poison apparatus and internal structure of the ovipositor (Quicke \& van Achterberg 1990, Quicke et al. 1992a, 1992b), the subfamily status of this group was restored again.

In the Palaearctic Region, the subfamily Rhyssalinae includes the genera Rhyssalus Haliday, Oncophanes Foerster, Dolopsidea Hincks, Pseudobathystomus Belokobylskij, Lysitermoides van Achterberg (described originally from the Nearctic Region), and Tobiason Belokobylskij (Belokobylskij 2004). Recently, two new genera from this subfamily were described from the Australasian Region - Anachyra van Achterberg and Rhyssaloides Belokobylskij (van Achterberg 1995, Belokobylskij 1999). It is possible that the Australasian genera Dorycto- morpha Ashmead, Caenopachyella Szépligeti and Metaspathius Brues are also members of this subfamily (Belokobylskij et al. 2004).

The generic position of Rogas (Colastes) funestus Haliday, 1836, the type species of the genus Pseudobathystomus, has been unclear for many years. It was included in the genera Colastes Haliday, Bathystomus Foerster (synonym of Opius Wesmael) and Rhysipolis Foerster (Shenefelt 1975). Study of the description and material from England of $C$. funestus has shown that is distinct from the above listed genera. As a result, the new genus Pseudobathystomus Belokobylskij was described with type species $R$. (C.) funestus (Belokobylskij 1986); the holotype (ठ) kept in National Museum of Ireland in Dublin was found later (van Achterberg 1997). Oncophanes tobiasi Zaykov [female, "Bulgaria, ex coll. Zaykov, RMNH Leiden 1991", "17.4.1966, Cernootchane, Kzh. (= Rhodopi Mts, near Kurdzhali), A Germanov", "Holotypus Oncophanes tobiasi + , Zaykov, 1980", "Oncophanes sp. n., Tobias det. 1978”, "Pseudobathy- 
stomus tobiasi (Zaykov), det. Belokobylskij, 1985"; holotype currently in the Nationaal Natuurhistorisch Museum of Leiden], described from Bulgaria (Zaykov 1983), was recently transferred to this genus (Belokobylskij 1986). An additional species of Pseudobathystomus, $P$. vernalis Belokobylskij, was described from the Russian Far East (Sakhalin I. and Primorskiy Kray) (Belokobylskij 1994).

Pseudobathystomus is a sister group of the genus Oncophanes. The main differences between these genera are listed below.

A new species of the genus Pseudobathystomus, namely $P$. macaronesius sp. n., from the Canary Islands, is described in this paper. Diagnostic characters for this species are (1) radial cell of fore wing open apically (metacarpus and third abscissa of radial vein unsclerotized apically at short distance), (2) pterostigma long and narrow, (3) antennal segments slender, and (4) mesoscutum mostly glabrous. These characters are phylogenetically significant and indicate the isolated position of this species within genus Pseudobathystomus. As result, a new subgenus, Atlantobathystomus subgen. n., is described.

Hosts of Pseudobathystomus are known only for P. funestus that attacks larvae of the Oecophoridae moths (Shenefelt 1975). It is possible that similar hosts (larvae of moths) will be discovered for other species of this genus (including $P$. macaronesius sp. n.).

The terms for wing venation are those defined by Belokobylskij and Tobias (1998). The following abbreviation are used: $\mathrm{POL}$ - postocellar line; OOL - ocular-ocellar line; Od - maximum diam- eter of lateral ocellus; ZISP - Zoological Institute, Russian Academy of Sciences (St.Petersburg, Russia); DABH - Department of Applied Biology, the University of Helsinki (Finland).

\section{Systematic section}

Genus Pseudobathystomus Belokobylskij, 1986

Atlantobathystomus subgen. n.

Type species: Pseudobathystomus macaronesius sp. n.

Description. Head transverse (dorsal view). Temple long. Occipital carina fused below with hypostomal carina, complete dorsally. Malar suture absent. Antennae slender, with long and slender segments. First flagellar segment longer than second segment. Apical segment pointed apically, without spine. Mesosoma short and high. Sides of pronotum with deep oblique and finely crenulate median furrow. Mesoscutum glabrous for the most part. Notauli narrow, deep and complete. Sternauli short and rather deep. Propodeum with distinctly marginate areas; areola rather large. Pterostigma narrow, almost as long as metacarpus. Radial cell open apically; metacarpus and third abscissa of radial vein unsclerotized apically at short distance. First abscissa of radial vein longer than maximum width of pterostigma. Second radiomedial cell long. Recurrent vein of hind wing present, but weakly sclerotized. Tergites of metasoma behind first one rather soft and smooth. Ovipositor long, longer than metasoma.

Etymology. From "Atlantic ocean" referring
1. Third-sixth or fourth-sixth metasomal tergites soft and deformed in dry dead specimens

2. At least basal part of second tergite sculptured

3. Ovipositor not serrate apico-ventrally

4. Ovipositor sheath short, distinctly shorter than mesosoma

5. Metascutum laterally densely sculptured

6. Sternauli sculptured
Third-sixth metasomal tergites distinctly sclerotized and not deformed in dry dead specimens

Second tergite usually entirely smooth

Ovipositor sparsely serrate apico-ventrally Ovipositor sheath long, not or sometimes slightly shorter than mesosoma Metascutum usually laterally smooth Sternauli almost entirely smooth 

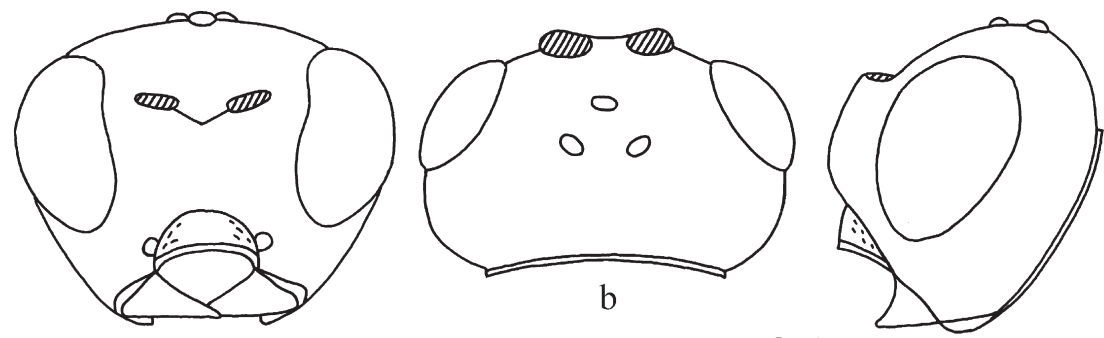

a
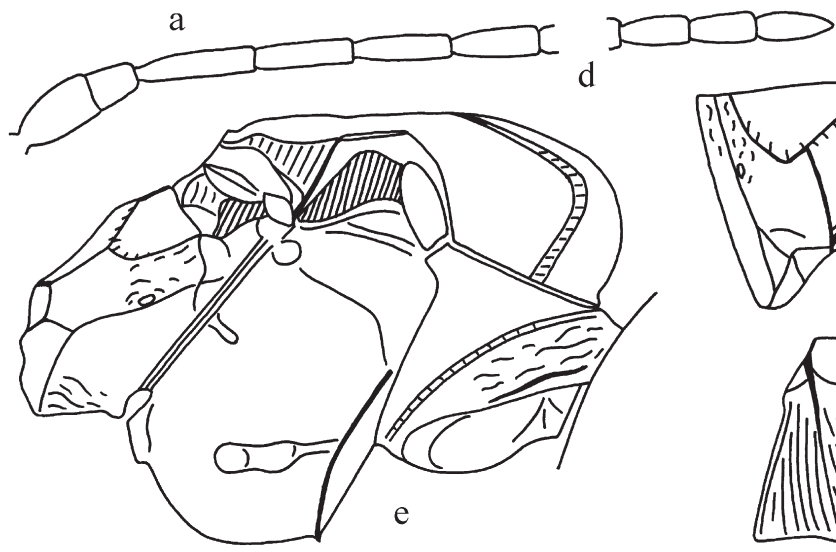

c

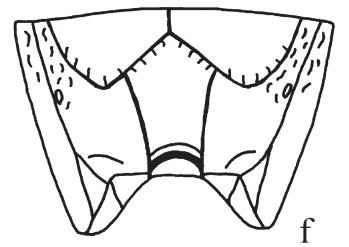

Fig. 1. Pseudobathystomus (Atlantobathystomus) macaronesius sp. n., holotype. - a. Head, frontal aspect. - b. Head, dorsal aspect. - c. Head, lateral aspect. -d. Basal and apical segments of antenna. - e. Mesosoma, lateral aspect. - f. Propodeum, dorsal aspect. - g. First metasomal tergite. h. Hind tibia. - i. Hind femur. -j. Fore wing. $-k$. Hind wing.

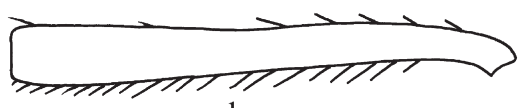

$\mathrm{h}$

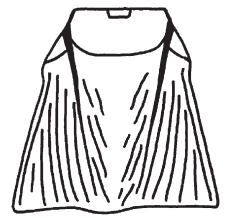

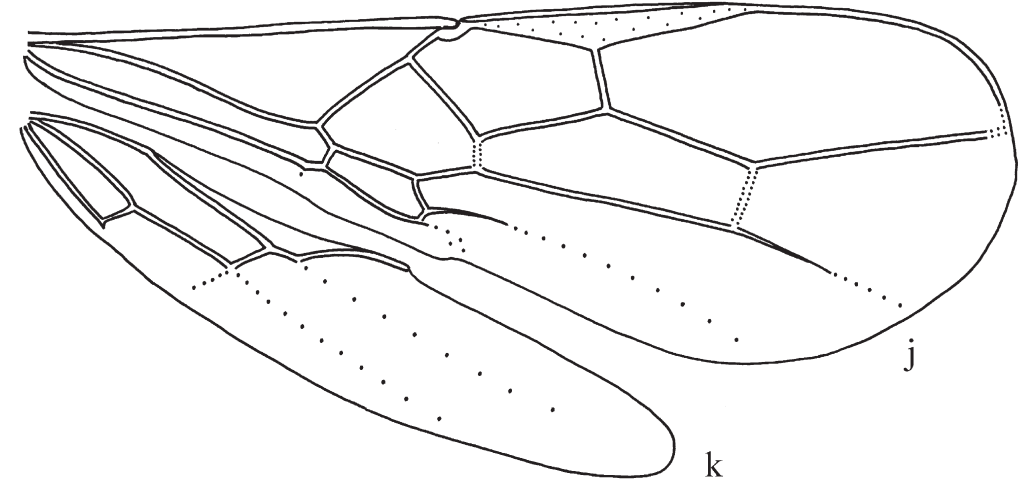

to the geographic locality of the Tenerife Island and the generic name "Bathystomus".

\section{Pseudobathystomus} macaronesius sp. n. (Fig. 1)

Material. Holotype, + (DABH), "Canary Islands, Tenerife, Anaga, El Bailadero, 13.12.1997, M. Koponen leg”. Paratype $1 \hat{\jmath}$, with same label as holotype (ZISP).

Description. Female. Body length $1.7 \mathrm{~mm}$; fore wing length $2.1 \mathrm{~mm}$.
Head width 1.7 times its median length (dorsal view), 1.2 times width of mesoscutum. Head behind eyes (dorsal view) almost parallel-sided anteriorly, strongly roundly narrowed posteriorly. Transverse diameter of eye almost equal to length of temple. Ocelli rather small, in triangle with base 1.1 times its sides, posterior ocelli situated on level of posterior margin of eye. POL 1.5 times Od, 0.5 times OOL. Eye glabrous, 1.4 times as high as broad. Malar space height 0.3 times height of eye, 0.8 times basal width of mandible. 
Face width equal to height of eye and 1.2 times height of face and clypeus combined. Clypeal suture distinct. Hypoclypeal depression round, its width equal to distance from edge of depression to eye, 0.4 times width of face. Occipital carina below fused with hypostomal carina. Third segment of labial palpus strongly reduced, almost indistinct.

Antennae rather slender, almost filiform, but weakly thickened apically, 20-segmented. Scapus almost twice as long as its maximum width, 1.6 times as long as pedicel. First flagellar segment about 4.0 times as long as its apical width, 1.1 times as long as second segment. Penultimate segment 2.2 times as long as wide, 0.6 times as long as first segment, 0.9 times as long as apical segment; the latter pointed apically and without spine.

Mesosoma length 1.4 times its height. Mesoscutum very highly and almost perpendicularly raised above pronotum. Notauli entirely deep, narrow, complete, smooth. Prescutellar depression rather deep, wide, with weak median carina, smooth, 0.3 times as long as scutellum. Scutellum convex, without lateral carinae. Subalar depression very shallow, wide, smooth. Sternauli shallow anteriorly and deep posteriorly, oblique, straight, but weakly curved in posterior half, smooth, almost connected with prepectal carina anteriorly, running along anterior half of lower part of mesopleura.

Fore wing 2.7 times as long as its maximum width. Pterostigma narrow, wedge-shaped, 6.5 times as long as wide. Radial cell open apically; third radial abscissa and metacarpus apically unsclerotized at short distance. Radial vein arising weakly before middle of pterostigma. Radial cell not shortened. Metacarpus as long as pterostigma. First radial abscissa 1.3 times as long as maximum width of pterostigma. Second radial abscissa about 3.0 times as long as first abscissa, 0.65 times as long as the straight third abscissa, 1.4 times as long as first radiomedial vein. Second radiomedial cell long, 3.2 times as long as its maximum width, 2.8 times as long as brachial cell. Recurrent vein postfurcal. Distance from nervulus to basal vein almost equal to nervulus length. Brachial cell distinctly widened distally. Hind wing 4.7 times as long as wide. First abscissa of costal vein 0.65 times as long as second abscissa. First abscissa of mediocubital vein 1.1 times as long as second abscissa. Recurrent vein almost straight, short, interstitial, and unsclerotized.

Hind femur about 5.0 times as long as wide. Hind tarsus almost as long as hind tibia. Hind basitarsus 0.65 times as long as second-fifth segments combined. Second tarsal segment 0.4 times as long as basitarsus, almost as long as fifth segment (without pretarsus).

Metasoma as long as mesosoma. First tergite with small dorsope, with small spiracular tubercles in basal $1 / 3$, almost linearly widened from base to apex. Maximum width of first tergite 1.8 times its minimum width, equal to its length. Second suture shallow, straight and complete. Median length of second tergite about 0.5 times its basal width, 1.5 times length of third tergite. Ovipositor long; its sheath 1.2 times as long as metasoma or mesosoma, 1.4 times as long as hind tibia, 0.4 times as long as fore wing.

Sculpture and pubescence. Head entirely smooth. Sides of pronotum smooth at most part, narrowly rugulose posteriorly. Mesonotum and mesopleura smooth, mesoscutum with 2 short striae medioposteriorly. Metapleura mostly smooth, rugose posteriorly. Propodeum smooth for the most part, partly with short rugae along carinae, with distinctly marginate areas, areola large and wide, pentagonal; basal carina short, 0.8 times as long as fork of areola. All legs smooth. First tergite with rather distinct, almost complete, weakly convergent posteriorly dorsal carinae, striate, but narrowly smooth on median part. Rest of tergites smooth. Vertex with short, sparse, almost adpressed setae. Mesosoma mostly glabrous, short setae present marginally and along notauli. Mesopleura glabrous, with very sparse setae only in lower $1 / 3$. Hind tibia dorsally with short dense and semi-erect setae.

Colour. Body black, mesosoma anterolaterally and mesoscutum medioposteriorly light reddish brown. Second and basal half of third tergites dirty yellowish brown. Antenna light reddish brown to reddish brown in basal half, almost black in apical half. Palpi and tegulae yellow. Legs yellow, apical 1/4 of hind tibia and all tarsi distinctly infuscate. Ovipositor sheath brown, black in apical $1 / 3$. Fore wing very faintly infuscate. Pterostigma greyish yellow. 
Male. Body length $1.7 \mathrm{~mm}$; fore wing length $1.9 \mathrm{~mm}$. First flagellar segment of antenna 3.6 times as long as its apical width. Penultimate segment 2.4 times as long as wide, 0.7 times as long as first segment, almost equal to apical segment. Length of mesosoma 1.45 times its height. Pterostigma 6.3 times as long as maximum width, a little shorter than metacarpus. Second radiomedial cell 3.0 times as long as maximum width, 3.0 times as long as brachial cell. First metasomal tergite narrow, weakly widened apically, with distinct spiracular tubercles, 1.3 times as long as apical width; its apical width 1.5 times basal width. Median length of second tergite 0.7 times its basal width. Basal carina of propodeum short, 0.4 times as long as fork of areola. Mesosoma entirely black. Tegulae brownish yellow. Otherwise similar to female.

\section{A key to the subgenera and species of the genus Pseudobathystomus Belokobylskij}

1. Radial cell open apically; metacarpus and third abscissa of radial vein unsclerotized apically at short distance. Pterostigma long and narrow, almost as long as metacarpus. First abscissa of radial vein longer than maximum width of pterostigma. Antenna slender and with long segments. Mesoscutum glabrous at most part. (Atlantobathystomus subgen. n.). Body length $1.7 \mathrm{~mm}$. - Canary Is.

P. (A.) macaronesius sp. n.

- Radial cell closed apically; metacarpus and third abscissa of radial vein sclerotized apically. Pterostigma shorter and wide, distinctly shorter than metacarpus. First abscissa of radial vein shorter than maximum width of pterostigma. Antenna thick and with short segments. Mesoscutum entirely densely setose. (Subgenus Pseudobathystomus Belokobylskij, s. str.)

2. Ovipositor sheath short, $0.65-0.7$ times as long as metasoma, 0.8 times as long as hind tibia, $0.25-0.3$ times as long as fore wing. Mesoscutum brownish red. Body length 3.0 $3.3 \mathrm{~mm}$. - England, Poland, Bulgaria

$$
\text { P. (P.) tobiasi (Zaykov) }
$$

- Ovipositor sheath long, 1.0-2.0 times as long as metasoma, 1.2-2.0 times as long as hind tibia, $0.4-0.65$ times as long as fore wing. Mesoscutum dark brown or brow, rarely (pale form) light brown, very rarely brownish red

3. Ovipositor sheath distinctly longer than metasoma, about twice as long as hind tibia, 0.55-0.65 times as long as fore wing. Pterostigma of male not largened. Second metasomal tergite medially weakly sculptured. Carinae of propodeal areola distinctly separated anteriorly. Body length 2.2-2.5 mm. - Russia (Primorskiy Terr., Sakhalin I.)

$P$. (P.) vernalis Belokobylskij

- Ovipositor sheath shorter than or subequal to metasoma, 1.1-1.5 times as long as hind tibia, 0.4-0.45 times as long as fore wing. Pterostigma of male largened. Second metasomal tergite entirely smooth. Carinae of propodeal areola usually fused or weakly separated anteriorly. Body length 2.1-3.1 mm. - England, France, Spain, Sweden, Germany, Finland, Lithuania, Belarus, Czech Republic, Hungary, Moldova, Ukraine, Russia (Voronezh Prov., Tuva, Primorskiy Terr., Sakhalin I., Kuril Is.)

$$
\text { P. (P.)funestus (Haliday) }
$$

Acknowledgements. We wish to express our sincere thanks to Dr. C. van Achterberg (Leiden, The Netherlands) for the loan of type material. We also thank Dr. P. Marsh (North Newton, KS, USA) for comments on the manuscript.

\section{References}

Achterberg, C. van 1995: Generic revision of the subfamily Betylobraconinae (Hymenoptera: Braconidae) and other groups with modified fore tarsus. - Zool. Verh. Leiden. 298: 3-242.

Achterberg, C. van 1997: Revision of the Haliday collection of Braconidae (Hymenoptera). - Zool. Verh. Leiden. 314: 3-115.

Belokobylskij, S. A. 1986: A new genus of the braconid wasps from supertribe Exothecidii (Hymenoptera, Braconidae). - Entomol. Obozr. 45: 780-783. [In Russian].

Belokobylskij, S. A. 1994: Review of the braconid wasps of the subfamilies Doryctinae and Exothecinae (Hymenoptera, Braconidae) of the Far East, Eastern Siberia and neighbouring territories. - Trudy "Daurski" 
Natural Reserve 3: 5-77. [In Russian].

Belokobylskij, S. A. 1999: New genera of the subfamilies Rhyssalinae, Exothecinae and Gnamptodontinae (Hymenoptera, Braconidae) from the Old World. Zoosyst. Ross. 8: 155-169.

Belokobylskij, S. A. 2004: Taxonomic reclassification of the East Asian species of the genus Oncophanes Foerster (Hymenoptera: Braconidae, Rhyssalinae). Proc. Russ. Entomol. Soc. 75: 106-117.

Belokobylskij, S. A., Iqbal, M. \& Austin, A. D. 2004: Systematics, distribution and diversity of the Australasian doryctine wasps (Hymenoptera: Braconidae: Doryctinae). - Records S. Austral. Mus. Monograph Series 8: 1-150.

Foerster, A. 1862. Synopsis der Familien und Gattungen der Braconen. - Verhandl. Naturhist. Ver. Preuss. Reinlande und Westphalens 19: 226-288.

Quicke, D. L. J. \& Achterberg, C. van 1990: Phylogeny of the subfamilies of the family Braconidae (Hymenoptera: Ichneumonoidea). — Zool. Verh.. Leiden 258: 1-95.

Quicke, D. L. J., Ficken, L. C. \& Fitton, M. G. 1992a: New diagnostic ovipositor characters for doryctine wasps (Hymenoptera, Braconidae). - J. Nat. Hist. 26: 10351046.

Quicke, D. L. J., Tunstead, J., Falco, J. V. \& Marsh, P. M. 1992b:Venom gland and reservoir morphology in the Doryctinae and related braconid wasps (Insecta, Hymenoptera, Braconidae). —Z Zool. Scripta 21: 403416.

Shenefelt, R. D. 1975: Exothecinae, Rogadinae. Braconidae 8. - Hymenopterorum Catalogus 12: 11151262.

Zaykov, A. N. 1983: Oncophanes tobiasi sp. n. (Hymenoptera, Braconidae), a new species from Bulgaria. - Acta Zool. Bulg. 22: 59-60. 